\title{
About the Chemical Evolution of dSphs (and the peculiar Globular Cluster $\omega$ Cen)
}

\author{
Andrea Marcolini ${ }^{1}$ and Annibale D'Ercole ${ }^{2}$ \\ ${ }^{1}$ Centre for Astrophysics, University of Central Lancashire, \\ Preston, Lancashire, PR1 2HE, United Kingdom \\ email: amarcolini@uclan.ac.uk \\ ${ }^{2}$ INAF, Osservatorio Astronomico di Bologna, \\ via Ranzani 1, 40127 Bologna, Italy \\ email: annibale.dercole@bo.astro.it
}

\begin{abstract}
We present three dimensional hydrodynamical simulations aimed at studying the dynamical and chemical evolution of the interstellar medium (ISM) in isolated dwarf spheroidal galaxies (dSphs). This evolution is driven by the explosion of Type II and Type Ia supernovae, whose different contribution on both the dynamics and chemical enrichment is taken into account. Radiative losses are effective in radiating away the huge amount of energy released by $\mathrm{SNe}$ explosions, and the $\mathrm{dSph}$ is able to retain most of the gas allowing a long period $(\geqslant 2-$ 3 Gyr) of star formation, as usually observed in this kind of galaxies. We are able to reproduce the stellar metallicity distribution function (MDF) as well as the peculiar chemical properties of strongly O-depleted stars observed in several dSphs. The model also naturally predicts two different stellar populations, with an anti-correlation between $[\mathrm{Fe} / \mathrm{H}]$ and velocity dispersion, similarly to what observed in the Sculptor and Fornax dSphs. These results derive from the inhomogeneous pollution of the SNe Ia, a distinctive characteristic of our model. We also applied the model to the peculiar globular cluster $(\mathrm{GC}) \omega$ Cen in the hypothesis that it is the remnant of a formerly larger stellar system, possibly a dSph.
\end{abstract}

Keywords. hydrodynamics, methods: numerical, stars: abundances, ISM: evolution, ISM: abundances, galaxies: dwarf, (Galaxy:) globular clusters: individual ( $\omega$ Cen)

\section{Introduction}

Due to their proximity, the galaxies of the Local Group (see Mateo 1995 and Geisler et al. 2007 for a review) offer a unique opportunity to study in details their structural, dynamical and chemical properties and to test different theories of galaxy formation.

Owing to their low metallicity and lack of neutral hydrogen, it was initially believed that dSphs are relatively simple objects whose ISM is completely removed by SN II explosions after a very short intense star formation period (e.g. Dekel \& Silk 1986). Doubts about this picture come from the high resolution spectroscopy of several dSphs showing a wide range in metallicity. For instance, Shetrone et al. (2001) have observed stars in Draco and Ursa Minor with values of $[\mathrm{Fe} / \mathrm{H}]$ in the range $-3 \leqslant[\mathrm{Fe} / \mathrm{H}] \leqslant-1.5$. The same authors also found that their observed dSphs have $[\alpha / \mathrm{Fe}]$ abundances that are 0.2 dex lower than those of Galactic halo field stars at the same metallicity. This suggests that the bulk of the stars in these systems formed in gas self polluted by SNe II as well as SNe Ia and that the star formation (SF) must continue over a relatively long timescale in order to allow a sufficient production of iron by SNe Ia (Ikuta \& Arimoto 2002, Lanfranchi \& Matteucci 2004, Marcolini et al. 2006; but see also Recchi et al. 2007, Salvadori et al. 2008). 

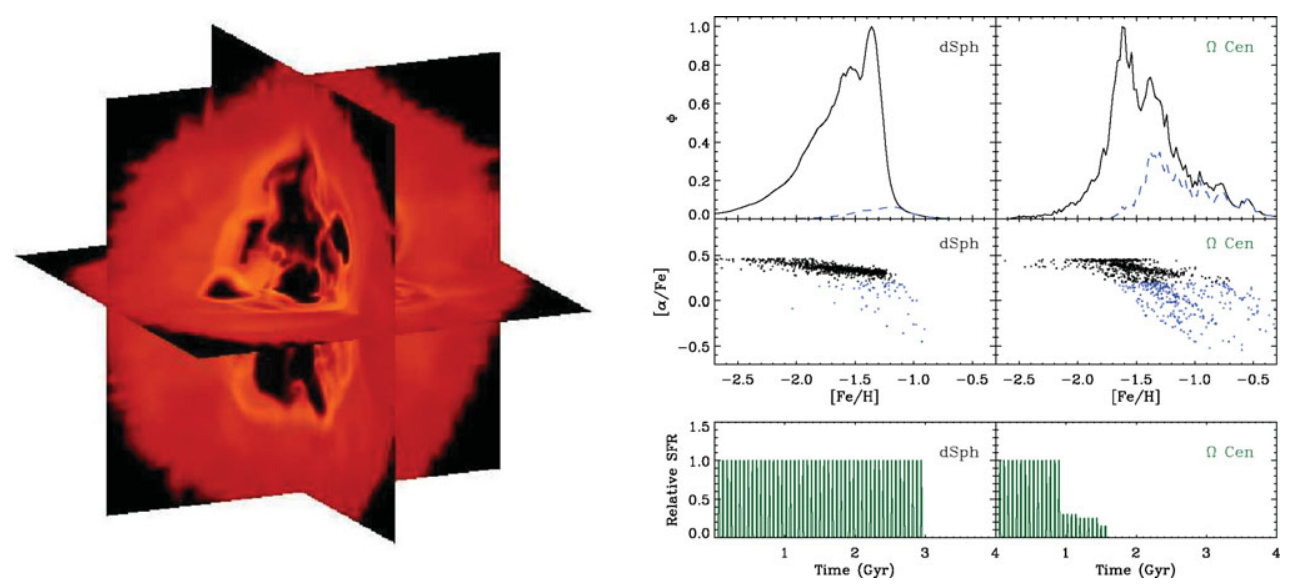

Figure 1. Left: distribution of the logarithm of the density in three orthogonal planes at $\sim 400$ Myr after the beginning of the simulation. Note the inner bubble carved by the SNe explosions and the shocks propagating outward. Right: $[\mathrm{Fe} / \mathrm{H}]$ distribution of the models resembling the Draco dSph (left panels) and the $\omega$ Cen globular cluster (right panels) together with the corresponding $[\alpha / \mathrm{Fe}]-[\mathrm{Fe} / \mathrm{H}]$ diagrams; the blue line and blue dots represent stars with $[\alpha / \mathrm{Fe}] \leqslant 0.2$ (i.e. affected by SNe Ia inhomogenous pollution, see text). The lower panels represent the assumed SFH for the two models.

A complex star formation history ( $\mathrm{SFH})$ is further suggested by several facts: $i$ ) isolated low mass dSphs such as Phoenix (Young et al. 2007) and Leo T (de Jong et al. 2008) were able to form stars up to 100 Myr ago; $i i$ ) the SFHs of dwarf galaxies are strongly dependent on their local environment, the fraction of passively evolving galaxies dropping from $\sim 70 \%$ in dense environments, to zero in the rarefied field (Haines et al. 2007 ); iii) dwarf ellipticals and dSphs cluster around the dominant spirals galaxies, while gas rich star forming dwarf Irregulars are found at larger distances (van den Bergh 1994). These points highlight the role of the environment (tidal interaction/ram pressure stripping), and disfavors a scenario in which the evolution is due uniquely to internal processes.

Here we briefly report some results obtained by Marcolini et al. (2006, 2007, 2008) with a model which turns to be consistent with many properties of the Draco dwarf and, with minimal assumptions, to the chemical properties of the peculiar system $\omega$ Centauri (Marcolini et al. 2007), which is believed to be the remnant of an ancient dSph.

\section{2. dSphs Model}

Let us consider the reference model of Marcolini et al. (2006) which, although employed to study the general characteristics of dSphs, is tailored to explore the evolution of the Draco dSph. The simulation starts with the ISM in hydrostatic equilibrium in the extended dark matter halo potential well. The amount of initial gas corresponds to the cosmological baryonic fraction of the dark matter halo $\left(\mathrm{M}_{\mathrm{ISM}}=0.18 \mathrm{M}_{\mathrm{DM}}\right)$. The SFH is given a priori, assuming that stars form in a sequence of 50 instantaneous bursts separated in time by $60 \mathrm{Myr}$ (see Fig. 1). We also assume that SNe II explode at a constant rate for $30 \mathrm{Myr}$ after the occurrence of each starburst, while the SNe Ia rate follows the prescription of Matteucci \& Recchi (2001). Each SN explosion is stochastically placed into the galaxy according to its radial probability $P(r)=M_{\star}(r) / M_{\star, t o t}$, where $M_{\star}(r)$ and $M_{\star, t o t}$ are the nowadays radial stellar mass profile and total stellar mass, respectively (cf. Marcolini et al. 2006 for more details). 


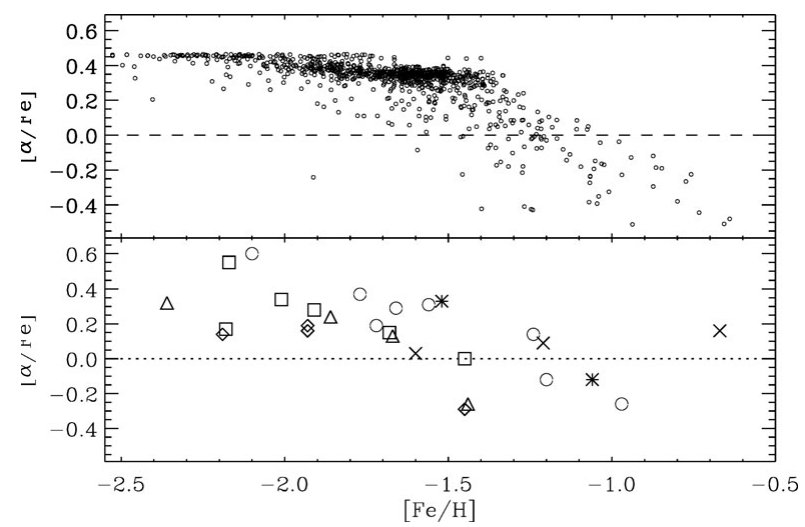

Figure 2. Abundance ratio $[\alpha / \mathrm{Fe}]$ versus $[\mathrm{Fe} / \mathrm{H}]$ for 500 sampled stars of the reference model at $t=2$ Gyr, compared to a dataset of 28 dSph stars (Draco: triangles ; Fornax: crosses; Leo I: asterisks; Sextans: diamonds; Sculptor: circles; Ursa Minor: squares) collected from the literature (see Marcolini et al. 2008 for details).

Although the total energy released by the SNe II explosions is larger than the binding energy of the ISM, efficient radiative losses enable the galaxy to retain most of its gas, which thus remains available for the aforementioned prolonged SFH. The burst of SNe II associated with each stellar burst pushes the bulk of the ISM to the outskirt of the galaxy (see Fig. 1, left panel). Once the explosions cease $(\sim 30 \mathrm{Myr}$ after each star burst episode), the ISM flows back towards the center of the galaxy; when the next burst occurs, the gas is pushed outwards again. This oscillatory behavior leads to a rather efficient and homogeneous pollution of the ISM by the SNe II ejecta. We note that as the SFH has been fixed a priori, it has no direct relation to the gas reservoir within the galaxy. However, stars do form during the quiescent periods between bursts, when the gas has "settled", and an a posteriori consistency for the SFH is recovered. For example a similar periodic ISM behaviour was recovered in similar low-mass galaxies simulations performed by Stinson et al. (2007). We point out that, while the observed dSphs are deprived of gas, the galaxy in the present model can not expel the bulk of its ISM by internal mechanisms. Following the discussion given in the Introduction, an external cause, such as the interaction with the Milky Way (Mayer et al. 2006), must be invoked at some evolutive stage of the galaxy to get rid of its ISM.

Given their lower rate, SNe Ia do not significantly affect the general hydrodynamical behavior of the ISM, but their role is relevant for the chemical evolution of the stars. Because of their longer evolutionary timescales, SN Ia progenitors created in previous starbursts continue to explode during the subsequent quiescent periods, when the gas is flowing back into the central region. During these periods the higher ambient gas density (together with the lower SNe Ia explosion rate) cause the SNe Ia remnants to be isolated from one another, forming chemically inhomogeneous regions (we refer to these regions as "SNe Ia pockets"). These pockets are "washed out" by successive phases of expansion and collapse of the ISM, due to the effects of SNe II, but new pockets form during the quiescent phases between consecutive starbursts. At odds with the ejecta of SNe II, SN Ia debris are rich in iron and deficient in $\alpha$-elements. Thus, stars forming in the SN Ia pockets possess lower $[\alpha / \mathrm{Fe}]$ and higher $[\mathrm{Fe} / \mathrm{H}]$ ratios than those formed elsewhere.

\subsection{Comparison with Local dSphs}

As stressed above, the reference model discussed in Marcolini et al. (2006) was tailored to fit the Draco galaxy. In Fig. 1 we show the MDF of this model at the end of the 


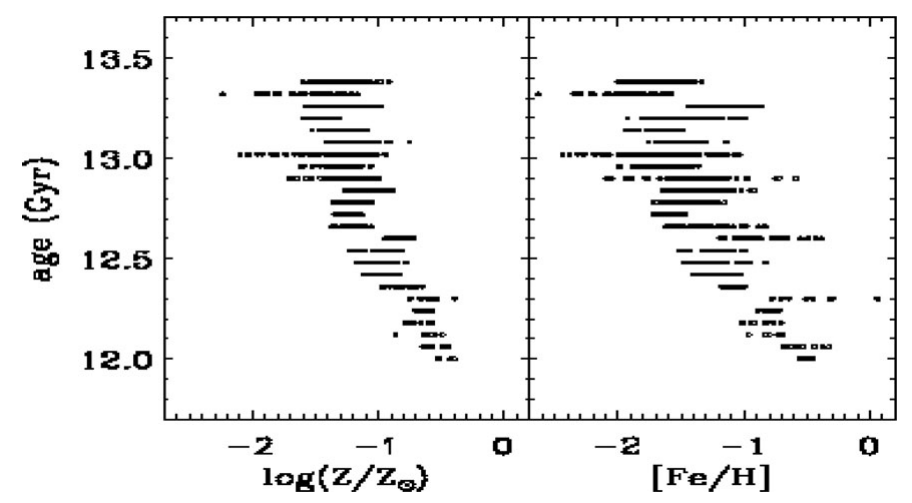

Figure 3. Age- $\mathrm{Z}$ and age- $[\mathrm{Fe} / \mathrm{H}]$ distributions of 1000 sampled stars for the $\omega$ Cen model. Note the metallicity spread among coeval stars.

simulation (3 Gyr). The maximum value of the distribution occurring at $[\mathrm{Fe} / \mathrm{H}] \sim-1.5$ and the mean value of $\langle[\mathrm{Fe} / \mathrm{H}]\rangle=-1.65$ with a spread of $\sim 1.5$ dex are compatible with observations (Bellazzini et al. 2002). The agreement is larger at the high metallicity tail of the distribution which, in our model, is shaped by the stars formed in the SN Ia pockets.

As discussed above, the SNe Ia are also responsible for the $[\alpha / \mathrm{Fe}]$ spread present at larger values of $[\mathrm{Fe} / \mathrm{H}]$ in the observed dSphs, as shown in Fig. 2 (lower panel). From this figure it is also apparent that the most metal poor stars have $[\alpha / \mathrm{Fe}] \simeq 0.5$, typical of pure SN II enrichment, while a decrement of $\sim 0.2$ dex in the plateau is achieved at higher metallicities. This drop occurs in our simulations after $t \sim 2.0-3.0$ Gyr to allow the cumulative effect of SNe Ia to be appreciable (top panel of Fig. 2). We thus conclude that, at least in our models, only a prolonged star formation history $(>2.0 \mathrm{Gyr})$ can account for the chemical differences between the Galactic halo and the dSphs as shown by Shetrone et al. (2001). Such a long SFH for dSphs is also found in the chemical models by Fenner et al. (2006) who are unable to reproduce the Ba/Y ratio unless stars formed over an interval long enough for the low-mass stars to pollute the ISM with $s$-elements.

The inhomogeneous pollution by SNe Ia discussed above is particularly important in the central galactic region, where the SN Ia rate is greater, and the density of the ambient gas is higher. This naturally accounts for a radial segregation of Fe-rich stars in the central regions of dSphs. As these stars in our model are also $\alpha$-depleted, a similar radial segregation of $\alpha$-poor stars should be observed to test our model. We finally stress that our model envisages a central depression in the radial distribution of the stellar velocity dispersion (Marcolini et al. 2008). This naturally entails two different stellar populations with an anti-correlation between $[\mathrm{Fe} / \mathrm{H}]$ and velocity dispersion, which has been observed in the case of the Sculptor dSph (Tolstoy et al. 2004) and the Fornax dSph (Battaglia et al. 2006).

\section{2. $\omega$ Cen}

The stellar system $\omega$ Cen (NGC 5139) is unique among Galactic star clusters in terms of its structure, kinematics, and stellar content. It is the only known GC showing a clear $[\mathrm{Fe} / \mathrm{H}]$ spread spanning the metallicity range $-1.6<[\mathrm{Fe} / \mathrm{H}]<-0.6$ (Norris et al. 1996, Johnson et al. 2008). Recent photometric surveys have revealed the presence of multiple sequences in its color-magnitude diagram (CMD), indicating a complex star formation history (e.g. Sollima et al. 2005). It is suggested that $\omega$ Cen is the nucleus of a larger stellar system, possibly a dwarf galaxy, that lost most of its stars and gas in the interaction with the Milky Way 10 Gyr ago (e.g. Bekki \& Norris 2006). In this 
framework Marcolini et al. (2007) focused on the evolution of the central region of their dSph model, where the inhomogeneous pollution of the SNe Ia is particularly effective.

In Fig. 1 the MDF within the inner 90 pc is plotted assuming a total SFH lasting $\sim 1.5$ Gyr as shown in the same Figure (see Marcolini et al. 2007 for more details and about the assumptions used for this model). The MDF shows a bimodal structure similar to that observed in $\omega$ Cen (e.g. Norris et al. 1996), with a maximum at $[\mathrm{Fe} / \mathrm{H}]=-1.6$ and a secondary peak at $[\mathrm{Fe} / \mathrm{H}] \sim-1.3$ accounting for $\sim 25 \%$ of the cluster's stellar content. Comparing the $[\alpha / \mathrm{Fe}]-[\mathrm{Fe} / \mathrm{H}]$ diagram of this model with the one typical of dSphs (c.f. Fig. 1), it is possible to note the much larger number of $\alpha$-depleted stars (see also the blue line in the MDF which represents stars with $\alpha / \mathrm{Fe} \leqslant 0.2)$. The diagram is in reasonable agreement with the findings of Pancino et al. (2002) and Origlia et al. (2003), who find that while the metal-poor and intermediate-metallicity stellar populations of $\omega$ Cen have the expected $\alpha$-element overabundance observed in halo and GC stars $(\langle[\alpha / \mathrm{Fe}]\rangle \simeq$ $0.3-0.4)$, the most metal-rich population $([\mathrm{Fe} / \mathrm{H}] \sim-0.6)$ shows a significantly lower $\alpha$ enhancement $(\langle[\alpha / \mathrm{Fe}]\rangle \simeq 0.1)$.

Given the distinctive role of the SN Ia pollution, the $[\mathrm{Fe} / \mathrm{H}]$ content of this system is not simply proportional to the metal content and a large spread in the age-metallicity relation is always present (see Fig. 3). These peculiarities have important consequences on the cluster CMD. In fact, they reduce by a factor of $40 \%$ the large (and still unexplained) helium overabundance usually invoked to account for the anomalous position of the blue main sequence observed in $\omega$ Cen.

\section{References}

Battaglia G. et al., 2006, A\&A, 459, 423

Bekki, K. \& Norris, J. E., 2006, ApJL, 637, L109

Bellazzini M., Ferraro F. R., Origlia L., Pancino E., Monaco L., \& Oliva E., 2002, AJ, 124, 3222 Dekel A. \& Silk J., 1986, ApJ, 303, 39

de Jong et al., 2008, astro-ph/08014027

Fenner, Y., Gibson, B. K., Gallino, R., \& Lugaro, M., 2006, ApJ, 646, 184

Geisler, D., Wallerstein, G., Smith, V. V., \& Casetti-Dinescu, D. I., 2007, PASP, 119, 939

Haines C., Gargiulo A., La Barbera F., Mercurio A., Merluzzi, \& Busarello, 2007, MNRAS, 381,7

Ikuta, C. \& Arimoto, N., 2002, A\&A, 391, 55

Johnson, C. I., Pilachowski, C. A., Simmerer, J., \& Schwenk, D., astro-ph/08042607

Lanfranchi G. A. \& Matteucci F., 2004, MNRAS, 351, 1338

Marcolini A., D'Ercole A., Brighenti F., \& Recchi S., 2006, MNRAS, 371, 643

Marcolini A., Sollima A., D'Ercole A., Gibson B. K., \& Ferraro F. R., 2007, MNRAS, 382, 443

Marcolini A., D'Ercole A., Battaglia G., \& Gibson B. K, 2008, MNRAS, 386, 2173

Mateo M. L., 1998, ARA\&A, 36, 435

Matteucci, F. \& Recchi, S., 2001, ApJ, 558, 351

Mayer L., Mastropietro C., Wadsley J., Stadel J., \& Moore B., 2006, MNRAS, 369, 1021

Norris J. E., Freeman K. C., \& Mighell K. J., 1996, ApJ, 462, 241

Origlia L., Ferraro F. R., Bellazzini M., \& Pancino E., 2003, ApJ, 591, 916

Pancino E., Pasquini L., Hill V., Ferraro F. R., \& Bellazzini M., 2002, ApJ, 568, L101

Recchi, S., Theis, C., Kroupa, P., \& Hensler, G, 2007, A\%A, 470, 5

Salvadori, S., Ferrara, A., \& Schneider, R., 2008, MNRAS, 386, 348

Shetrone M. D., Côté P., \& Sargent W. L. W., 2001, ApJ, 548, 592

Sollima A., Ferraro F. R., Pancino E., \& Bellazzini M., 2005, MNRAS, 357, 265

Stinson, G. S., Dalcanton, J. J., Quinn, T., Kaufmann, T., \& Wadsley, J., 2007, ApJ, 667, 170

Tolstoy E., et al. 2004, ApJ, 617, L119

van den Bergh S., 1994, ApJ, 428, 617

Young L. M., Skillman E. D., Weisz D. R., \& Dolphin A. E., 2007, ApJ, 659, 331 